\title{
Computed tomography coronary angiography - past, present and future
}

Pei Ing $\underline{\text { Ngam }}^{1}$, MBBS, FRCR, Ching Ching $\underline{\text { Ong }}^{1}$, MBBS, FRCR, Ping Chai ${ }^{2}$, MBBS, MRCP, Siong Sung $\underline{\text { Wong}}^{2}$, MD, MRCP, Chong Ri Liang ${ }^{1}$, MSc, Lynette Li San $\underline{\text { Teo }}^{1}$, MBChB, FRCR

\begin{abstract}
Computed tomography coronary angiography (CTCA) is a robust and reliable non-invasive alternative imaging modality to invasive coronary angiography, which is the reference standard in evaluating the degree of coronary artery stenosis. CTCA has high negative predictive value and can confidently exclude significant coronary artery disease (CAD) in low to intermediate risk patients. Over the years, substantial effort has been made to reduce the radiation dose and increase the cost efficiency of CTCA. In this review, we present the evolution of computed tomography scanners in the context of coronary artery imaging as well as its clinical applications and limitations. We also highlight the future directions of CTCA as a one-stop non-invasive imaging modality for anatomic and functional assessment of CAD.
\end{abstract}

Keywords: cardiac imaging, computed tomography, coronary angiography, coronary artery disease, CT scanners

\section{INTRODUCTION}

Cardiovascular disease (CVD) is the leading cause of death globally. Data from the World Health Organization shows that an estimated 17.7 million people died from CVD in 2015, representing $31 \%$ of all global deaths. Of these deaths, an estimated 7.4 million were due to coronary heart disease. ${ }^{(1)}$ The Singapore Heart Foundation estimates that 16 people die from CVD every day in Singapore. In 2016, CVDs accounted for 29.5\% of all deaths in Singapore. ${ }^{(2)}$

Invasive coronary angiography (ICA) is the gold standard for evaluation of the coronary artery lumen, in view of its high spatial and temporal resolution. In the last three decades, computed tomography coronary angiography (CTCA) has emerged as a robust and reliable non-invasive imaging modality for the assessment of coronary artery disease (CAD). The high negative predictive value of normal CTCA images can effectively exclude significant $C A D$, thereby avoiding the need for further imaging tests and reducing the need for ICA as the first-line investigation in patients with low to intermediate risk of CAD. ${ }^{(3)}$

In this review, we discuss the evolution of CTCA and review the current and future applications of CTCA in the assessment of CAD.

\section{THE PAST}

Prior to the 1980s, anatomic imaging of coronary arteries was performed directly with cardiac catheterisation. Indirect assessment of the haemodynamic significance of coronary artery lesions was performed via stress nuclear medicine studies and echocardiography. The era of computed tomography (CT) imaging of the coronary arteries began in the late 1990s, with the widespread introduction of single-slice helical CT systems. Prior to that, non-helical CT, electron beam CT and dynamic spatial reconstructor technologies provided an important foundation for cardiac imaging. However, these early scanners had limited spatial and contrast resolution, limiting their use to assessment of the myocardium, cardiac chambers and gross coronary calcification in clinical practice. ${ }^{(4)}$ With the introduction of multi-slice helical CT scanners, starting from the four-slice single-source CT scanners in 1998 to the 64-slice single-source CT scanners in 2004 (Table I); there was significant improvement in spatial and temporal resolution. This allowed for evaluation of clinically relevant branches of the coronary tree. ${ }^{(5)}$

However, the assessment of CAD with CTCA remained difficult in certain patient groups. First, in patients with high or irregular heart rate, the assessment of small mobile coronary arteries was limited by excessive coronary artery motion. These patients required beta-blockade to lower their heart rate to improve image quality. This translated to additional logistic requirements, as patients had to wait for the beta-blockade to take effect to lower the heart rate for an optimal scan. Their vital signs also had to be monitored regularly by nursing staff. Also, in patients with coronary stents or severely calcified arteries, resultant blooming and beam-hardening artefacts limited the accurate assessment of CAD on CTCA.

These limitations of CTCA were overcome with the introduction of first-generation dual-source CT (DSCT) scanners in 2006 and the subsequent development of wide-detector CT scanners in 2008. The first-generation DSCT scanner featured two X-ray tubes coupled with two separate arrays of detectors, allowing further improvement in temporal resolution. This enabled faster image acquisition with fewer motion artefacts and improved image quality. A study assessing the diagnostic accuracy of DSCT for the evaluation of CAD in a population with extensive coronary calcifications without heart rate control showed overall sensitivity, specificity, positive and negative predictive values of $96.4 \%, 97.5 \%, 85.7 \%$ and $99.4 \%$, respectively. ${ }^{(6)}$ Wide-detector CT scanners, namely 128-, 256- and 320-slice CT scanners,

${ }^{1}$ Department of Diagnostic Imaging, National University Hospital, ${ }^{2}$ Department of Cardiology, National University Heart Centre Singapore, Singapore Correspondence: Dr Lynette Teo Li San, Senior Consultant, Department of Diagnostic Imaging, National University Hospital, 5 Lower Kent Ridge Road, Singapore 119074. lynette_Is_teo@nuhs.edu.sg 
Table I. Chronology of X-ray computed tomography (CT) related to cardiac imaging. ${ }^{(4)}$

\begin{tabular}{|llll|}
\hline Yr & CT scanner & Slice per revolution and no. of X-ray tube** & Gantry rotation speed (s) \\
\hline 1975 & EBCT & - & - \\
\hline 1980 & DSR & - & - \\
\hline 1989 & SHCT & 1 slice per revolution & $0.8-1.0$ \\
\hline 1992 & 2 MSCT & 2 slices per revolution & $0.5-0.8$ \\
\hline 1998 & 4 MSCT & 4 slices per revolution & $0.38-0.5$ \\
\hline $2001-2002$ & 8-32 MSCT & 8, 16 and 32 slices per revolution & $0.33-0.42$ \\
\hline 2004 & 64 MSCT & 64 slices per revolution & 0.33 \\
\hline $2005-2006$ & DSDECT & 2 X-ray tubes coupled with 2 detector arrays & $0.27-0.35$ \\
\hline $2007-2008$ & 320 and 256 MSCT & 320 and 256 slices per revolution & $0.35-0.4$ \\
\hline $2008-2013$ & SSDECT & 1 X-ray tube with fast kVp switching coupled with 1 detector or 1 X-ray & 0.275 \\
\hline 2012 & & tube coupled with dual-layer detector & 640 slices per revolution \\
\hline
\end{tabular}

*Where applicable. CT: computed tomography; DSDECT: dual-source dual-energy CT; DSR: dynamic spatial reconstructor; EBCT: electron beam CT; MSCT: multi-slice CT; SHCT: single helical CT; SSDECT: single-source dual-energy CT

enabled greater coverage per gantry rotation, making whole-heart coverage possible in a single gantry rotation. These CT scanners had superior spatial and temporal resolution and could achieve improved image quality even in patients who were difficult to scan with older-generation CT scanners. Cost savings occurred due to increased patient turnover, improved patient satisfaction and decreased report turnaround time. ${ }^{(7)}$

\section{THE PRESENT}

Despite promising results from these advances and technical improvements in CT technology, a major disadvantage of CTCA is the ionising radiation exposure and potential risk of radiationrelated malignancy. Although there is no strong evidence of a causal relationship between CTCA-related ionising radiation exposure and cancer risks, a few epidemiological studies show higher cancer risks in subjects who received cardiac imagingequivalent radiation doses. ${ }^{(8,9)}$

The National Academies' Biological Effects of lonizing Radiation 7th Report (BEIR VII Phase 2) provides a framework for estimating cancer risks associated with radiation exposure from CTCA, using data from atomic bomb survivor studies and a few medical and occupational radiation studies. This report supports the linear no-threshold model, which states that (a) the risk of cancer is directly proportional to the radiation dose, and (b) there is no dose below which there is no risk. ${ }^{(10)}$ Nevertheless, this model carries certain assumptions, bringing with it uncertainties regarding radiation risk estimates. Also, several societies and scientific bodies, such as the American Association of Physicists in Medicine, stated that the "risks of medical imaging at effective doses below $50 \mathrm{mSv}$ for single procedures or $100 \mathrm{mSv}$ for multiple procedures over short time periods are too low to be detectable and may be non-existent" ${ }^{(11,12)}$

From the late 2000s to the present, further technological advancements have brought about even faster CT scanners, ranging from 640-slice dynamic volume CT scanners to thirdgeneration DSCT scanners and spectral CT. With each successive generation of $\mathrm{CT}$ scanners, radiation dose, contrast dose and patient turnaround time for CTCA have plummeted, while image quality has improved. This has resulted in a plethora of publications showing the promising diagnostic and prognostic value of CTCA in patients with suspected or known CAD using a relatively low radiation dose. Given the above, CTCA has become an attractive non-invasive first-line imaging modality for CAD.

The average radiation dose for a combined CT coronary calcium score and CTCA for an adult patient used to range between six and $20 \mathrm{mSv}$ (equivalent to 300-1000 chest radiographs) in the past using traditional retrospective cardiac-gating techniques. The subsequent introduction of prospective cardiac-gating in CTCA reduced the radiation dose by approximately $70 \%{ }^{.13)}$ Using the latest 640-slice CT scanner or a third-generation DSCT scanner, the radiation dose can now be further reduced to submillisievert doses (equivalent to $<50$ chest radiographs) for both $\mathrm{CT}$ coronary calcium score and CTCA. ${ }^{(14)}$ These faster scanners have also made it possible to reduce the contrast load from an average of $80 \mathrm{~mL}$ to $35 \mathrm{~mL}$, thus reducing the risk of contrast-induced nephropathy. ${ }^{(15,16)}$

These improvements in radiation dose, contrast dose and patient turnaround time can be attributed to technological advances in hardware and software, which are discussed below.

\section{Hardware \\ $X$-ray tube}

The evolution from a static X-ray tube to a rotating X-ray tube, coupled with improvements in its features such as higher heat capacity and cooling rate, has increased the efficiency of CT scanners, allowing for higher gantry speeds. ${ }^{(17)}$

The introduction of the DSCT scanner, which uses two X-ray tubes, has resulted in greater efficiency in obtaining a full data set, with each X-ray tube only requiring a $90^{\circ}$ rotation, further shortening image acquisition time and reducing radiation dose. The third-generation DSCT scanner offers substantially increased tube power at low tube potential, thus enabling significant radiation dose reduction. ${ }^{(17)}$ 


\section{Number of detectors and gantry speed}

Newer-generation CT scanners have increased the number of detectors and faster X-ray tube(s) rotation (also known as gantry speed). This also allows for faster image acquisition with reduction in the radiation dose. ${ }^{(17)}$

\section{Pitch}

The CT scanner table moves after each gantry rotation so that the next segment of the heart can be imaged. Pitch is defined as the ratio of table distance travelled in one gantry rotation to the X-ray beam width. ${ }^{(17)}$ Therefore, faster table movement shortens the scan acquisition time, leading to lower radiation dose.

\section{Software}

\section{Image postprocessing techniques}

Filtered back projection is a reconstruction technique that was used when CT was first developed in the 1970s. Although it is highly optimised and extremely quick, it results in increased image noise.

A robust technique of image postprocessing known as iterative reconstruction was widely used for clinical purposes in the 2000s. Compared with standard analytical reconstruction methods, iterative reconstruction produces equivalent signal-to-noise ratios at lower radiation doses without any loss of spatial resolution. ${ }^{(14)}$

\section{Postprocessing workstations}

Advancements in postprocessing workstations and software have allowed easier and faster evaluation of the coronary arteries on CTCA images.

In recent years, spectral imaging using single-source dualenergy CT scanners has emerged as a promising technique. In spectral CT, multiple spectrally distinct attenuation data sets are obtained from the same scan, thus enabling material composition analysis. This allows the detection and potential quantification of several distinct signals, which provide (a) more accurate coronary plaque characterisation; (b) optimal evaluation of the coronary artery lumen in patients with calcified plaques and coronary stents by reducing blooming artefact; and (c) assessment of myocardial perfusion. ${ }^{(18,19)}$

\section{CLINICAL APPLICATIONS}

The updated 2016 National Institute for Health and Care Excellence guidelines recommend CTCA as a first-line investigation in all patients with suspected stable CAD in view of its clinical efficacy and cost-effectiveness. ${ }^{(20)}$ The Society of Cardiovascular Computed Tomography Guidelines Committee has published appropriate use criteria for CTCA to guide clinicians. $^{(21)}$

\section{Diagnostic value}

\section{Assessment of native coronary arteries}

CTCA is widely used for detection of CAD in (a) symptomatic patients with low or intermediate pretest probability of CAD; (b) patients with low or intermediate pretest probability of CAD and newly diagnosed heart failure without known ischaemic heart disease; and (c) preoperative cardiac assessment in patients with low or intermediate pretest probability of CAD. The expansion of multi-slice CT (MSCT) scanners from 64-slice to 128-, 256-, 320- and 640-slice systems has enabled accurate assessment of coronary artery stenosis severity and atherosclerotic plaque composition. One meta-analysis showed the excellent diagnostic accuracy of CTCA in the detection of coronary plaques compared to intravascular ultrasonography (IVUS) as the reference standard, with a sensitivity of 0.90 and a specificity of $0.92 .{ }^{(22)}$

Other than stable CAD, CTCA is also an alternative to ICA in patients with suspected acute coronary syndrome (ACS) who have low or intermediate pretest probability of CAD. Four randomised trials, CT-STAT, ${ }^{(23)}$ ACRIN-P, ${ }^{(24)}$ ROMICAT II ${ }^{(25)}$ and CT-COMPARE, ${ }^{(26)}$ have compared CTCA to the standard of care in the evaluation of over 3,000 low-to-intermediate risk patients with suspected ACS. These trials reiterate the well-established negative predictive value of CTCA and consistently demonstrate the safety of discharging patients with negative CTCA from emergency departments with very low rates $(<1 \%)$ of major adverse cardiovascular events (MACE). This has resulted in cost savings and greater efficiency by reducing time to discharge and length of hospital stay. However, in patients with high pretest probability of CAD, ICA should be considered as the first-line imaging modality due to the lower negative predictive value of CTCA in this group. ${ }^{(27)}$

\section{Post-revascularisation assessment Coronary artery bypass grafts}

The American Heart Association (AHA) guidelines state that CTCA may be reasonable for the assessment of grafts in patients with new or worsening symptoms not consistent with unstable angina. ${ }^{(28)}$ There is, however, insufficient evidence to suggest that CTCA is beneficial in asymptomatic patients. CTCA is found to have superior diagnostic accuracy in assessing coronary artery bypass grafts (CABG) than in native vessels, probably owing to the fact that these grafts are usually of larger calibre with lower propensity to develop calcified plaques. Given their relative distance from the heart, fewer motion artefacts are seen in grafts. Furthermore, studies show that CTCA is able to identify grafts that cannot be detected or accessed by ICA. ${ }^{(29)}$ However, the assessment of internal mammary artery and distal grafts can be more challenging due to artefacts caused by adjacent metal clips and their relatively smaller diameter.

A meta-analysis of 15 articles by Hamon et al shows the excellent diagnostic accuracy of CTCA in assessing grafts using 16- and 64- slice scanners, as evidenced by a sensitivity of $97.6 \%$, specificity of $96.7 \%$ and negative predictive value of $98.9 \%{ }^{(30)}$

\section{Coronary artery stents}

In contrast to $\mathrm{CABG}$, the evaluation of coronary stents using CTCA is more challenging owing to beam hardening and partial volume artefacts. Therefore, the AHA guidelines suggest using CTCA for assessment of coronary stent patency in patients with new or worsening symptoms not consistent with unstable angina. There 
is also insufficient evidence to suggest that CTCA is beneficial in asymptomatic patients. ${ }^{(28)}$

The positive predictive value of detecting in-stent restenosis using CTCA is low. The diagnostic accuracy of CTCA is better in stents with diameters $\geq 3 \mathrm{~mm}$ and with thinner struts $(<100 \mu \mathrm{m})$. $^{(31)}$ It may be improved by performing CTCA on symptomatic patients with a higher pretest probability or asymptomatic patients for evaluation of left main coronary artery stents. ${ }^{(21,32)}$

\section{Assessment of coronary artery anomalies}

CTCA is superior to ICA in assessing coronary artery anomalies, offering better anatomical depiction of coronary arteries and allowing anomalies of origin, course and termination to be established more clearly. This is particularly important in potentially life-threatening congenital anomalies such as the malignant course of an anomalous right coronary artery (RCA), in which the proximal RCA has an interarterial course between the aorta and main pulmonary artery. CTCA is also useful for accurate measurement of coronary artery origins, particularly for pre-procedural planning such as transcatheter aortic valve implantation or difficult coronary artery catheterisation.

\section{Prognostic value}

The presence, extent and severity of CAD on CTCA are independent predictors of future MACE. ${ }^{(33)}$ In a meta-analysis consisting of 32 relatively large studies with 41,960 patients, Habib et al studied the prognostic value of CTCA in patients with CAD. ${ }^{(34)}$ Their analysis showed that the rate of cardiac events was $0.04 \%$ in patients without CAD, $1.29 \%$ in patients with nonobstructive CAD and $6.53 \%$ in patients with obstructive CAD.

Coronary plaque composition is also an important prognostic determinant of CAD. Among all imaging techniques, IVUS, optical coherence tomography (OCT) and angioscopy provide the closest information to match the histopathology of atherosclerotic plaques. ${ }^{(35)}$ However, the widespread use of these modalities is limited by their invasive nature, high cost and inability to examine the entire coronary artery. CTCA is therefore an important noninvasive modality in coronary plaque characterisation, being able to identify vulnerable plaques that show the features of positive remodelling, low attenuation $(<30$ Hounsfield units [HU]), the napkin-ring sign and spotty calcification. A multicentre study to rule out myocardial infarction by cardiac CT (ROMICAT-II) showed that the presence of high-risk plaques on CTCA in patients with low to intermediate risk of CAD increases the likelihood of ACS independent of significant CAD and clinical risk assessment. ${ }^{(36)}$

In addition, thin-cap fibroatheroma (TCFA) is an independent predictor of future cardiac events. ${ }^{(37)}$ A TCFA is defined as a plaque containing a lipid core that occupies more than two quadrants of the cross-sectional vascular area $\left(>180^{\circ}\right)$ and $<65 \mu$ m cap thickness on OCT. It can be identified indirectly on CT using the napkin-ring sign, which demonstrates the presence of a ring-like attenuation in a cross-sectional area of the coronary artery. The napkin-ring sign may be a surrogate marker of TCFAs: in a study that compared OCT and CTCA, the sign was seen in $44 \%$ of the TCFA group compared to $4 \%$ of the non-TCFA group. ${ }^{(38)}$
Table II. Spatial, contrast and temporal resolution of CT and ICA.

\begin{tabular}{|llll|}
\hline Type & $\begin{array}{l}\text { Spatial } \\
\text { resolution }(\mathbf{m m})\end{array}$ & $\begin{array}{l}\text { Contrast } \\
\text { resolution }\end{array}$ & $\begin{array}{l}\text { Temporal } \\
\text { resolution }(\mathbf{m s})\end{array}$ \\
\hline CT & $0.5-0.625$ & Low to moderate & $83-135$ \\
\hline ICA & 0.16 & Moderate & $1-10$ \\
\hline
\end{tabular}

CT: computed tomography; ICA: invasive catheter angiography

\section{CHALLENGES \\ Radiation dose}

In recent years, the radiation dose associated with CTCA has received heightened attention. Manufacturers have made great strides in radiation dose reduction with hardware and software improvements. The mean effective dose for single-source MSCT coronary angiography (four- to 64-slice CT scanners) was between $6.0 \pm 2.8 \mathrm{mSv}$ and $11.8 \pm 5.9 \mathrm{mSv}$, and this has dropped to submillisievert levels (equivalent to $<50$ chest radiographs) for current third-generation DSCT and wide-detector CT scanners. ${ }^{(39,40)}$ Hence, it is now possible to produce diagnostic CTCA images with a very low radiation dose. This dose will only get lower with better scanners in the years ahead.

\section{Image quality}

The image reconstruction process is a fundamental determinant of image quality. As mentioned above, filtered back projection has traditionally been used in CT image reconstruction. Although it causes an increase in spatial resolution, it is directly associated with increased image noise and higher radiation exposure. Newer scanners employ various iterative reconstruction techniques to reduce quantum noise and artefacts, resulting in improved image quality.

\section{Temporal and spatial resolution}

In general, the spatial, contrast and temporal resolution of CT are inferior to that of catheter angiography (Table II). ${ }^{(41)}$ Widedetector MSCT and DSCT scanners have shorter gantry rotation times, allowing improvement in temporal resolution independent of the patient's heart rate. ${ }^{(42)}$ The best temporal resolution for a single source CT is $75 \mathrm{~ms}$, compared with $66 \mathrm{~ms}$ for a DSCT. However, the temporal resolution is still inferior to that of ICA. Therefore, heart rate control is crucial in CTCA examinations to reduce motion artefacts and maximise temporal resolution.

\section{Motion artefacts}

Motion artefacts are invariably caused by cardiac and breathing motion. These can be minimised by a few measures.

\section{Heart rate control}

Vigorous heart rate control using beta-blockers can significantly reduce motion artefacts and improve image quality. This is particularly important in CT scanners with relatively lower temporal resolution. Imaging specialists and clinicians should be aware of the contraindications of beta-blockers such as asthma, heart failure, severe aortic stenosis, obstructive hypertrophic cardiomyopathy, heart block and sick sinus syndrome without a permanent pacemaker. 


\section{Retrospective electrocardiography-triggered technique}

The retrospective electrocardiography (ECG)-triggered technique allows data to be acquired throughout the entire cardiac cycle. If the heart rate remains high and/or irregular with heart rate variability of more than five beats per minute despite betablockers, ${ }^{(43)}$ the retrospective ECG-triggered technique would be the method of choice to achieve high image quality. The main drawback of this technique is the higher radiation dose required, thus limiting its use.

\section{Hardware and software development}

Wide-detector CT scanners or DSCT scanners now allow the scanning of patients with higher and irregular heart rates with satisfactory image quality. The increased longitudinal scan coverage of wide-detector $\mathrm{CT}$ scanners and faster gantry rotation speed of DSCT scanners allow three-dimensional volumetric whole-heart imaging within one cardiac cycle.

\section{Breath-hold technique}

The technological advancements from 16- to 640-slice systems have progressed with improved longitudinal volume coverage, decreased gantry rotation time and smaller detector elements. These indirectly decrease scan time, resulting in significantly shorter breath-holds and, therefore, fewer motion artefacts related to poor breath-holding.

\section{Beam hardening and blooming artefacts}

Beam hardening is due to the attenuation of low-energy $\mathrm{X}$-rays by high-attenuating objects. The X-ray beam 'hardens' as the degree of attenuation distal to the attenuating structure decreases. In CTCA, this artefact occurs when high density structures, such as coronary artery calcium and small stents with a diameter of less than $3 \mathrm{~mm}$, occupy a portion of $\geq 1 \operatorname{voxel}(\mathrm{s})$. The resultant images demonstrate dark and bright streaks around the dense object. This artefact can be minimised with the application of sharper image filters, newer image postprocessing software or the use of dual energy imaging. ${ }^{(44)}$

Blooming artefacts are present when there is significant coronary artery calcification, resulting in oversized calcified plaques on the CT image with subsequent overestimation of luminal narrowing. At the same time, this limits the characterisation of vulnerable soft plaques. These artefacts can be minimised by the smaller voxel size produced by $\geq 64$-slice CT scanners. ${ }^{(45)}$ Some centres do not perform CTCA if the calcium score is greater than a predetermined threshold (typically Agatston score $>600-1,000)^{(46)}$ due to excessive blooming artefacts. However, current high-end scanners have hardware and software improvements to mitigate blooming artefacts.

\section{Stair-step artefact}

Stair-step artefacts occur when there are inconsistencies in volumetric datasets secondary to differences in R-R intervals over several cardiac cycles, especially in patients with high heart rates, heart rate variability and the presence of irregular or ectopic heart beats such as premature ventricular contractions and atrial fibrillation during image acquisition.
Good heart rate control using beta-blockers and reconstructing the dataset at different phases of the cardiac cycle are fundamental measures to minimise this artefact. The latest wide-detector CT and DSCT scanners allow image acquisition in a single heartbeat, and this can potentially eliminate these artefacts, even in patients with arrhythmias.

\section{FUTURE DIRECTIONS Functional assessment}

Conventional CTCA is limited to the anatomic depiction of coronary arteries and does not provide functional information on a coronary lesion. With technological advances, CTCA has the potential to become a single one-stop non-invasive study for both anatomic and functional information. Three techniques have been demonstrated to accurately detect vessel-specific ischaemia: non-invasive CT fractional flow reserve (CT-FFR); transluminal attenuation gradient (TAG); and CT perfusion imaging (CTP).

\section{Computed tomography fractional flow reserve}

Fractional flow reserve (FFR) is used along with ICA as the invasive reference standard for estimating ischaemia and to guide coronary revascularisation in cases with discrepancy between the degree of coronary stenosis and the presence of myocardial ischaemia.

In recent years, CT-FFR has emerged as a novel technology that enables determination of the haemodynamic significance of coronary lesions non-invasively. It also provides a 'virtual stenting' facility, whereby the physiological effect of alternative interventional strategies can be trialled in silico before treatment is delivered in vivo. This eliminates the need for invasive $\mathrm{FFR}$, ${ }^{(47)}$ reducing costs, radiation exposure and potential patient complications associated with ICA.

CT-FFR uses sophisticated computer algorithms based on computational fluid dynamics applied to pre-existing CTCA images, obviating the need for additional imaging, radiation dose or additional medications such as adenosine or other vasodilators. Currently, its clinical use is limited due to inconvenience and costs.

\section{Transluminal attenuation gradient}

TAG is defined as the linear regression coefficient between luminal attenuation and axial distance from the coronary ostium. It has the potential to assess the degree of coronary stenosis by evaluating the slope of decline in intraluminal contrast attenuation from the ostium to the distal coronary vessel.

TAG shows a sensitivity of $77 \%$, specificity of $74 \%$ and negative predictive value of $86 \%$ in detecting haemodynamically significant coronary stenosis using a retrospectively determined TAG cut-off of $-15.1 \mathrm{HU} / 10 \mathrm{~mm}$. The combined TAG and CTCA assessment has an area under the curve of $0.88 .{ }^{(48)}$

\section{Computed tomography perfusion imaging}

Myocardial perfusion imaging on CTCA requires the acquisition of images during the first pass of iodinated contrast from the arteries into the myocardium, where hypoperfusion is represented by hypo-attenuated areas. Myocardial CTP requires the acquisition 
of stress and rest images. The stress images allow for evaluation of myocardial perfusion after administration of coronary vasodilators such as adenosine, dipyridamole or regadenoson. Conventionally, stress CTP is performed first, followed by rest CTP. This is to avoid contamination of the myocardium by the contrast medium that is given during the first scan, which can potentially mask underlying myocardial ischaemia. The coupling of stress and rest CTP allows the reversibility of the myocardial defect to be determined.

A study by Magalhães at al showed that compared with CTCA alone, combined CTCA and myocardial CTP has higher specificity (CTCA alone: $54 \%$ vs. combination of CTCA and CTP: $73 \%$ ) and overall accuracy (CTCA alone: $69 \%$ vs. combination of CTCA and CTP: $75 \%)$. ${ }^{(49)}$ The major disadvantage of CTP is the higher radiation dose, with the mean radiation dose ranging from $9.2 \mathrm{mSv}$ to $12.5 \mathrm{mSv}$ using a 128 -detector DSCT. ${ }^{(50)}$ In addition, stress-rest CTP requires two boluses of contrast, which increases excretory burden, especially in patients with renal impairment.

\section{Coronary plaque characterisation}

Lesions resulting in ACS often have a large necrotic, lipid-rich core. Current scanners can differentiate between calcified plaques and non-calcified plaques (NCPs). However, the sub-classification of NCPs into lipid-rich and fibrous lesions remains challenging, as there is substantial overlap in plaque densities, preventing reliable sub-classification. New automated plaque quantification software tools, with scan-specific adaptive attenuation threshold settings, can potentially overcome some of these limitations and may improve CT number-based plaque component quantification. ${ }^{(22)}$

\section{Computer-aided diagnosis and artificial intelligence}

Computer-aided diagnosis is widely applied to assist physicians in the interpretation of medical images. The computer algorithm generally consists of image processing, image feature analysis and data classification via the use of tools such as artificial neural networks. ${ }^{(51)}$ These may be referred to as artificial intelligence (AI). $\mathrm{Al}$ is able to incorporate patients' demographic data, clinical presentation and laboratory results with radiology images for analysis and then highlight conspicuous sections, such as possible diseases.

In the foreseeable future, a combination of anatomic, functional and perfusional parameters, together with computerassisted diagnosis using $\mathrm{Al}$, will replace our current modus operandi of only anatomic evaluation of the coronary arteries.

\section{CONCLUSION}

CTCA is a robust and reliable non-invasive alternative imaging to ICA in evaluating CAD. It is especially useful in the evaluation of patients with low to intermediate pretest probability of CAD. Given the current advantages of CTCA, the challenge is for physicians to judiciously utilise this resource in order to keep healthcare costs in check.

Although there have been significant improvements in CT scanner hardware and post-processing software, resulting in CTCA images with high diagnostic quality, more research is required to resolve the limitations associated with CTCA. Continued improvement and refinement of current CT scanners is expected in the years to come. In the near future, CTCA will be a one-stop non-invasive imaging modality for both anatomic and functional assessment of CAD. This will further solidify its position as the modality of choice for diagnosis and prognostication of CAD, and additional stress-related or invasive procedures can be avoided.

\section{REFERENCES}

1. Färber D, Hahn H, Fendt-Klug T, Höpner F. [Thoracic trauma in childhood. Radiologic findings]. Radiologe 1995; 35:385-90. German.

2. Ministry of Health, Singapore. Principal causes of death. In: Singapore Heart Foundation [online]. Available at: http://www.myheart.org.sg/article/about-theheart-and-heart-disease/statistics/singapore/75. Accessed August 13, 2018.

3. Miller JM, Rochitte CE, Dewey M, et al. Diagnostic performance of coronary angiography by 64-row CT. N Engl J Med 2008; 359:2324-36.

4. Hurlock GS, Higashino H, Mochizuki T. History of cardiac computed tomography: single to 320-detector row multislice computed tomography. Int J Cardiovasc Imaging 2009; 25 Suppl 1:31-42

5. Meijboom WB, Meijs MF, Schuijf JD, et al. Diagnostic accuracy of 64-slice computed tomography coronary angiography: a prospective, multicenter, multivendor study. J Am Coll Cardiol 2008; 52:2135-44.

6. Scheffel H, Alkadhi H, Plass A, et al. Accuracy of dual-source CT coronary angiography: first experience in a high pre-test probability population without heart rate control. Eur Radiol 2006; 16:2739-47.

7. Min JK, Gilmore A, Budoff MJ, Berman DS, O'Day K. Cost-effectiveness of coronary CT angiography versus myocardial perfusion SPECT for evaluation of patients with chest pain and no known coronary artery disease. Radiology 2010; 254:801-8.

8. Shapiro BP, Mergo PJ, Snipelisky DF, Kantor B, Gerber TC. Radiation dose in cardiac imaging: how should it affect clinical decisions? AJR Am J Roentgenol 2013; 200:508-14

9. Einstein AJ. Effects of radiation exposure from cardiac imaging: how good are the data? J Am Coll Cardiol 2012; 59:553-65.

10. National Research Council. Health Risks from Exposure to Low Levels of lonizing Radiation: BEIR VII Phase 2. Washington, DC: The National Academies Press; 2006.

11. Zanzonico P, Dauer L, Strauss HW. Radiobiology in cardiovascular imaging. JACC Cardiovasc Imaging 2016; 9:1446-61.

12. Lam DL, Larson DB, Eisenberg JD, Forman HP, Lee Cl. Communicating potential radiation-induced cancer risks from medical imaging directly to patients. AJR Am J Roentgenol 2015; 205:962-70.

13. Kuchynka P, Lambert L, Černý V, et al. Coronary CT angiography. Cor Vasa 2015; 57:e425-32.

14. Halliburton SS, Abbara S, Chen MY, et al. SCCT guidelines on radiation dose and dose-optimization strategies in cardiovascular CT. J Cardiovasc Comput Tomogr 2011; 5:198-224.

15. Zhang F, Yang L, Song X, et al. Feasibility study of low tube voltage (80kVp) coronary $\mathrm{CT}$ angiography combined with contrast medium reduction using iterative model reconstruction (IMR) on standard BMI patients. Br J Radiol 2016; 89:20150766.

16. Raju R, Thompson AG, Lee K, et al. Reduced iodine load with CT coronary angiography using dual-energy imaging: a prospective randomized trial compared with standard coronary CT angiography. J Cardiovasc Comput Tomogr 2014; 8:282-8.

17. Ulzheimer S, Flohr T. Multislice CT: Current Technology and Future Developments. In: Reiser M, Becker C, Nikolaou K, Glazer G, eds. Multislice CT. Medical Radiology. Springer-Verlag Berlin Heidelberg, 2009: 3-23.

18. Danad I, Fayad ZA, Willemink MJ, Min JK. New applications of cardiac computed tomography: dual-energy, spectral, and molecular CT imaging. JACC Cardiovasc Imaging 2015; 8:710-23.

19. Rajiah P, Abbara S, Halliburton SS. Spectral detector CT for cardiovascular applications. Diagn Interv Radiol 2017; 23:187-93.

20. National Institute for Health and Clinical Excellence. Chest pain of recent onset: assessment and diagnosis of recent onset chest pain or discomfort of suspected cardiac origin. Updated November 2016. London: Royal College of Physicians (UK).

21. Taylor AJ, Cerqueira M, Hodgson JM, et al. ACCF/SCCT/ACR/AHA/ASE/ ASNC/NASCI/SCAI/SCMR 2010 appropriate use criteria for cardiac computed tomography. A report of the American College of Cardiology Foundation Appropriate Use Criteria Task Force, the Society of Cardiovascular Computed Tomography, the American College of Radiology, the American Heart Association, the American Society of Echocardiography, the American Society of Nuclear Cardiology, the North American Society for Cardiovascular Imaging, the Society for Cardiovascular Angiography and Interventions, and the Society for Cardiovascular Magnetic Resonance. J Cardiovasc Comput Tomogr 2010; 4:407.e1-33. 
22. Voros S, Rinehart S, Qian Z, et al. Coronary atherosclerosis imaging by coronary CT angiography: current status, correlation with intravascular interrogation and meta-analysis. JACC Cardiovasc Imaging 2011; 4:537-48.

23. Goldstein JA, Chinnaiyan KM, Abidov A, et al; CT-STAT Investigators. The CTSTAT (Coronary Computed Tomographic Angiography for Systematic Triage of Acute Chest Pain Patients to Treatment) trial. J Am Coll Cardiol 2011; 58:1414-22.

24. Litt HI, Gatsonis C, Snyder B, et al. CT angiography for safe discharge of patients with possible acute coronary syndromes. N Engl J Med 2012; 366:1393-403.

25. Hoffmann U, Truong QA, Schoenfeld DA, et al; ROMICAT-II Investigators. Coronary $\mathrm{CT}$ angiography versus standard evaluation in acute chest pain. N Engl J Med 2012; 367:299-308.

26. Hamilton-Craig C, Fifoot A, Hansen M, et al. Diagnostic performance and cost of CT angiography versus stress ECG--a randomized prospective study of suspected acute coronary syndrome chest pain in the emergency department (CT-COMPARE). Int J Cardiol 2014; 177:867-73.

27. Arbab-Zadeh A, Miller JM, Rochitte CE, et al. Diagnostic accuracy of computed tomography coronary angiography according to pre-test probability of coronary artery disease and severity of coronary arterial calcification. The CORE-64 (coronary artery evaluation using 64-row multidetector computed tomography angiography) International Multicenter Study. J Am Coll Cardiol 2012; 59:379-87.

28. Stirrup J, Velasco A, Hage FG, Reyes E. Comparison of ESC and ACC/AHA guidelines for myocardial revascularization. J Nucl Cardiol 2017; 24:1046-53.

29. Jabara R, Chronos N, Klein L, et al. Comparison of multidetector 64-slice computed tomographic angiography to coronary angiography to assess the patency of coronary artery bypass grafts. Am J Cardiol 2007; 99:1529-34.

30. Hamon M, Lepage O, Malagutti P, et al. Diagnostic performance of 16- and 64-section spiral CT for coronary artery bypass graft assessment: meta-analysis. Radiology 2008; 247:679-86.

31. Andreini D, Pontone G, Bartorelli AL, et al. Comparison of feasibility and diagnostic accuracy of 64-slice multidetector computed tomographic coronary angiography versus invasive coronary angiography versus intravascular ultrasound for evaluation of in-stent restenosis. Am J Cardiol 2009; 103:1349-58.

32. Kolh P, Windecker S, Alfonso F, et al. 2014 ESC/EACTS Guidelines on myocardial revascularization: the Task Force on Myocardial Revascularization of the European Society of Cardiology (ESC) and the European Association for Cardio-Thoracic Surgery (EACTS). Developed with the special contribution of the European Association of Percutaneous Cardiovascular Interventions (EAPCI). Eur J Cardiothorac Surg 2014; 46:517-92.

33. Nakazato R, Arsanjani R, Achenbach S, et al. Age-related risk of major adverse cardiac event risk and coronary artery disease extent and severity by coronary CT angiography: results from 15,187 patients from the International Multisite CONFIRM Study. Eur Heart J Cardiovasc Imaging 2014; 15:586-94.

34. Habib PJ, Green J, Butterfield RC, et al. Association of cardiac events with coronary artery disease detected by 64-slice or greater coronary CT angiography: a systematic review and meta-analysis. Int J Cardiol 2013; 169:112-20.

35. Tian J, Ren X, Vergallo R, et al. Distinct morphological features of ruptured culprit plaque for acute coronary events compared to those with silent rupture and thin-cap fibroatheroma: a combined optical coherence tomography and intravascular ultrasound study. J Am Coll Cardiol 2014; 63:2209-16.

36. Puchner SB, Liu T, Mayrhofer T, et al. High-risk plaque detected on coronary
CT angiography predicts acute coronary syndromes independent of significant stenosis in acute chest pain: results from the ROMICAT-II trial. J Am Coll Cardiol 2014; 64:684-92

37. Xie Y, Mintz GS, Yang J, et al. Clinical outcome of nonculprit plaque ruptures in patients with acute coronary syndrome in the PROSPECT study. JACC CardiovasC Imaging 2014; 7:397-405.

38. Kashiwagi $\mathrm{M}$, Tanaka A, Kitabata $\mathrm{H}$, et al. Feasibility of noninvasive assessment of thin-cap fibroatheroma by multidetector computed tomography. JACC Cardiovasc Imaging 2009; 2:1412-9.

39. Sabarudin A, Sun Z, Ng KH. A systematic review of radiation dose associated with different generations of multidetector CT coronary angiography. J Med Imaging Radiat Oncol 2012; 56:5-17.

40. Hell MM, Bittner D, Schuhbaeck A, et al. Prospectively ECG-triggered highpitch coronary angiography with third-generation dual-source CT at $70 \mathrm{kVp}$ tube voltage: feasibility, image quality, radiation dose, and effect of iterative reconstruction. J Cardiovasc Comput Tomogr 2014; 8:418-25.

41. Lin E, Alessio A. What are the basic concepts of temporal, contrast, and spatial resolution in cardiac CT? J Cardiovasc Comput Tomogr 2009; 3:403-8.

42. Alkadhi H, Scheffel H, Desbiolles L, et al. Dual-source computed tomography coronary angiography: influence of obesity, calcium load, and heart rate on diagnostic accuracy. Eur Heart J 2008; 29:766-76.

43. Earls JP. How to use a prospective gated technique for cardiac CT. J CardiovasC Comput Tomogr 2009; 3:45-51.

44. Song BG. Multidector CT imaging of coronary artery stent and coronary artery bypass graft. In: Baskot BG, eds. What We Should Know about Prevented, Diagnostic, and Interventional Therapy in Coronary Artery Disease. InTechOpen, 2013.

45. Brodoefel H, Burgstahler C, Tsiflikas I, et al. Dual-source CT: effect of heart rate, heart rate variability, and calcification on image quality and diagnostic accuracy. Radiology 2008; 247:346-55

46. Abbara S, Blanke P, Maroules CD, et al. SCCT guidelines for the performance and acquisition of coronary computed tomographic angiography: a report of the Society of Cardiovascular Computed Tomography Guidelines Committee: endorsed by the North American Society for Cardiovascular Imaging (NASCl). J Cardiovasc Comput Tomogr 2016; 10:435-49.

47. Morris PD, van de Vosse FN, Lawford PV, Hose DR, Gunn JP. "Virtual" (computed) fractional flow reserve: current challenges and limitations. JACC Cardiovasc Interv 2015; 8:1009-17.

48. Wong DT, Ko BS, Cameron JD, et al. Transluminal attenuation gradient in coronary computed tomography angiography is a novel noninvasive approach to the identification of functionally significant coronary artery stenosis: a comparison with fractional flow reserve. J Am Coll Cardiol 2013; 61:1271-9.

49. Magalhães TA, Kishi S, George RT, et al. Combined coronary angiography and myocardial perfusion by computed tomography in the identification of flowlimiting stenosis - the CORE320 study: an integrated analysis of CT coronary angiography and myocardial perfusion. J Cardiovasc Comput Tomogr 2015; 9:438-45.

50. Rossi A, Merkus D, Klotz E, et al. Stress myocardial perfusion: imaging with multidetector CT. Radiology 2014; 270:25-46.

51. Shiraishi J, Li Q, Appelbaum D, Doi K. Computer-aided diagnosis and artificial intelligence in clinical imaging. Semin Nucl Med 2011; 41:449-62. 